\title{
Taller de Inducción para la Instalación del CATI en las diferentes Facultades, Centros de Investigación, Laboratorios Especializados e IPS de UNAN - Managua
}

El día 8 de septiembre del 2015 se llevó a cabo el "Taller de Inducción para la Instalación del CATI en las diferentes Facultades, Centros de Investigación, Laboratorios e IPS de UNAN Managua", en el laboratorio 1102 de la facultad de Ciencias e Ingenierías.

\section{Objetivos del taller}

a. Potenciar el capital de conocimientos, mejorando el acceso a la bibliografía científica y tecnológica a docentes de la UNAN - Managua.

b. Fomentar los procesos de adopción de tecnología y servir de plataforma para incentivar los procesos de desarrollo.

c. Facilitar y apoyar (atención personalizada) a los usuarios en la búsqueda de información tecnológica disponible en:

i. base de datos de patentes;

ii. publicaciones electrónicas (científicas y técnicas) no relativas a las patentes;

iii. consulta "on line" de temas complementarios

d. Describir el proceso de innovación hasta su concesión de patente.

e. Describir el proceso de protección de la innovación por medio del registro de patentes o cualquier otra forma de protección de los derechos de propiedad intelectual relacionada con el producto.

f. Informar los procedimientos sobre la creación, protección, titularidad y gestión de los derechos de propiedad intelectual.

g. Compartir información sobre investigaciones científicas y técnicas de dominio público.

\section{Participantes}

1. Docentes de las diferentes Facultades de UNAN - Managua

2. Comisión de Innovación de UNAN Managua.

3. Representantes de los Centros de Investigación de la UNAN - Managua 
4. Funcionarios del MIFIC

\section{Programa}

\section{Primer Momento - Registro, Apertura, Objetivos y Conferencias}

Se inició con la inscripción de los participantes integrado por los Coordinadores de los CATI's en las Facultades y Centros de Investigación e IPS de la UNAN - Managua designados por los decanos y directores de los centros de investigación e IPS de la UNAN - Managua, para coordinar los centros de asistencia tecnológica.

Las palabras del Maestro Javier Pichardo Ramírez refiriéndose a la creación de los pilares bases de la protección de los productos intelectuales de UNAN - Managua, dirigidos por la dirección de Investigación de Grado y la Vicerrectorado de Investigación, y como la UNAN Managua articula los procesos de investigación, extensión y docencia en la culminación integrado.

En el taller de inducción se planteó las líneas principales de trabajo de los CATI’s y cómo ayudará al proceso de desarrollo de los prototipos de las invenciones creadas por los estudiantes y docentes, investigadores e innovadores de la UNAN - Managua, también se abordó sobre del uso de las bases de datos de patentes, utilizando la información y especificaciones generales de invenciones desarrolladas alrededor del mundo.

La Ponencia del Ing. Cesar Pereira, sobre el proceso de vinculación institucional de UNAN - Managua y MIFIC, como estrategia académica y profesional, para desarrollar la gestión de la Propiedad Intelectual e Industrial en UNAN - Managua, estrategia que fomenta los procesos de innovación y desarrollo de ciencia y tecnología en Nicaragua, cuya cuna son los docentes y estudiantes investigadores, innovadores y emprendedores. 


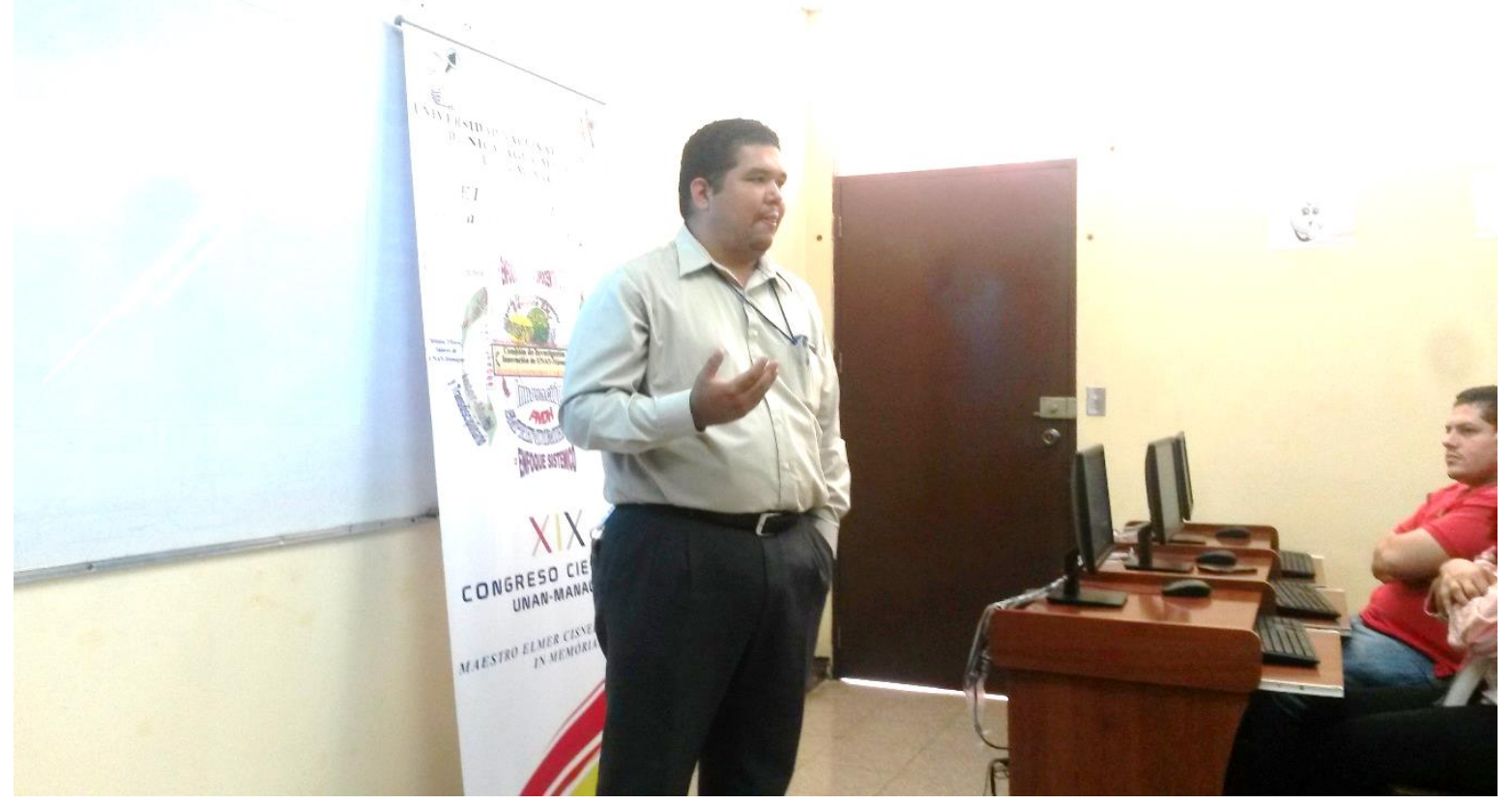

Figura 1. Ing. César Ponencia sobre el proceso de Vinculación de UNAN - Managua

El primer bloque del taller fue impartido por el Ing. Erick Zúñiga, dónde explico las leyes rectoras vigentes en Nicaragua para la protección de la propiedad intelectual de las creaciones intelectuales a nivel nacional. Aclaró el papel del CATI como medio de articulación de la innovación desde la universidad y de cómo la transferencia de tecnología ayuda a los países en vías de desarrollo para adaptar las tecnologías para mejorar la competencia nacional.

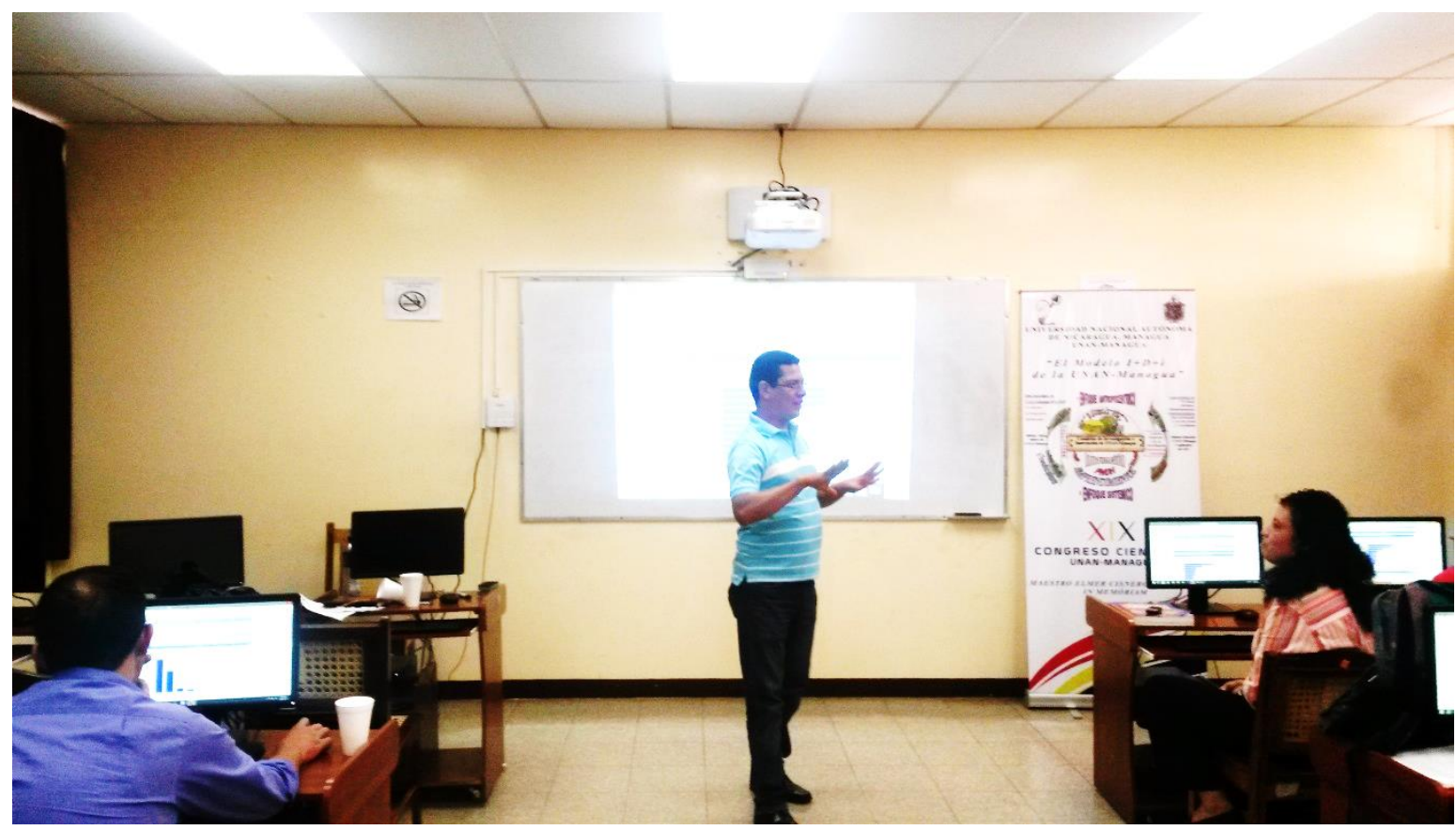


Figura 2. Ing. Erick Zúñiga, Funcionario del MIFIC - RPI.

El Ing. Maykol Salazar expuso sobre la instalación del CATI/UNAN - Managua y los avances concernientes acerca de la gestión administrativa de los procesos de articulación de los centros de Investigación, Facultades e IPS de la UNAN - Managua.

Posteriormente se detalló, las funciones de los coordinadores regionales en cada Facultad, Centro de Investigación e IPS encargados de los CATI, la población objetivo, los resultados esperados, usuarios finales, usuarios objetivos, usuarios a corto plazo, objetivos de la instalación del CATI/UNAN - Managua, Logros Esperados y los procesos de instalación del CATI central.

\section{Segundo Momento:}

El segundo momento comprende la explicación general del uso de la bases de datos para realizar las investigaciones sobre el estado de la técnica de la invención desarrollada, la posible estrategia de modificación de la descripción de la invención de la patente, registro de marcas y sobre la metodología de la descripción de marca, derechos de autor, derechos convexos y diseños industriales.

Ing. Wilmer Sequeira e Ing. Maykol Salazar, con el asesoramiento del Ing. Erick Zúñiga del RPI - MIFIC, apoyó el ejercicio sobre el uso de las bases de datos, para obtener información general de invenciones de acceso público y la adopción de tecnología para mejorar propuestas y lograr la competencia internacional. 


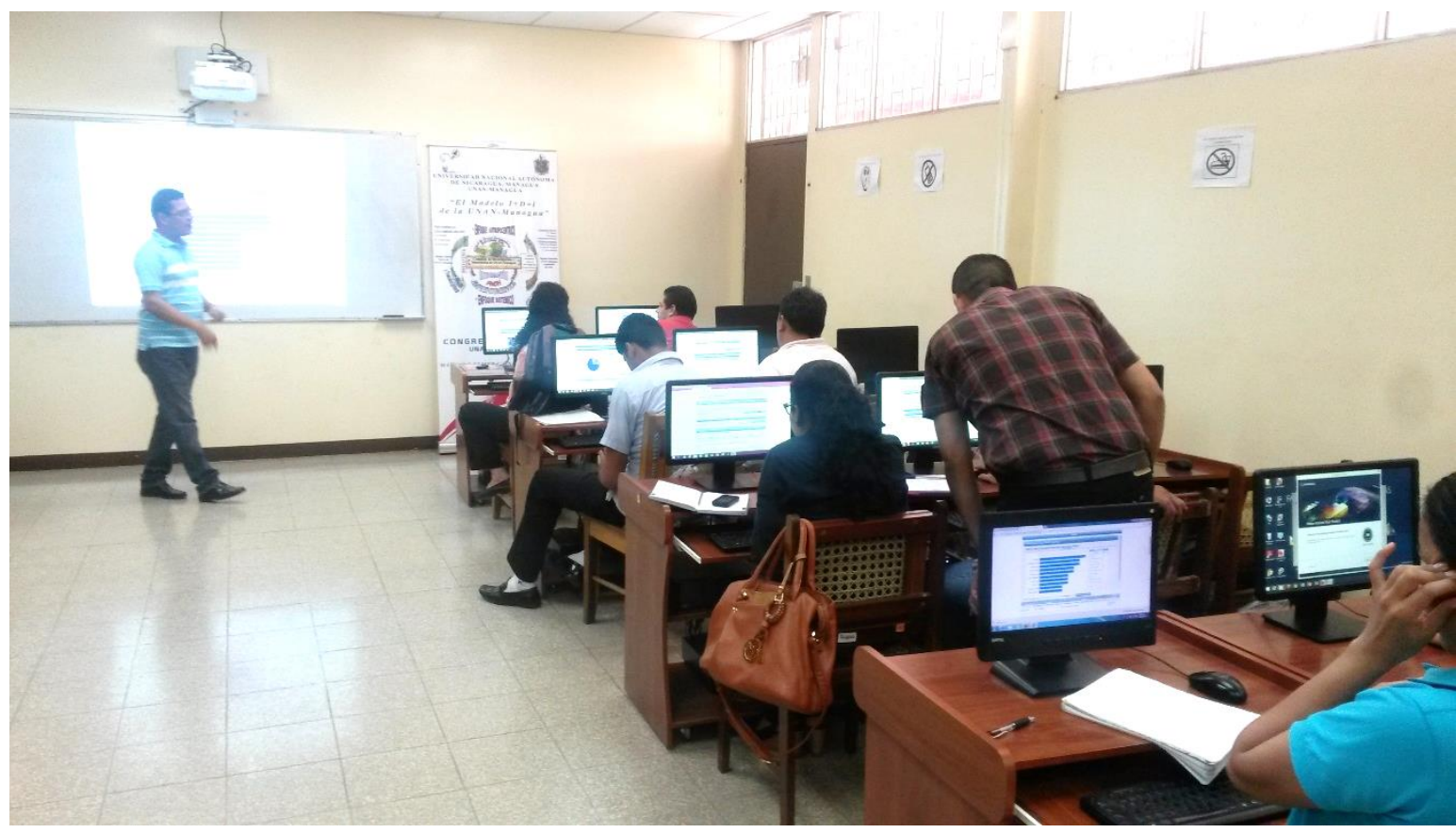

Figura 3. Eric Zúñiga, explicando el uso de la base de datos de PATENTSCOPE (Izquierda) y Asistencia Directa del Ing. Wilmer Sequeira Calero (Derecha, de pie).

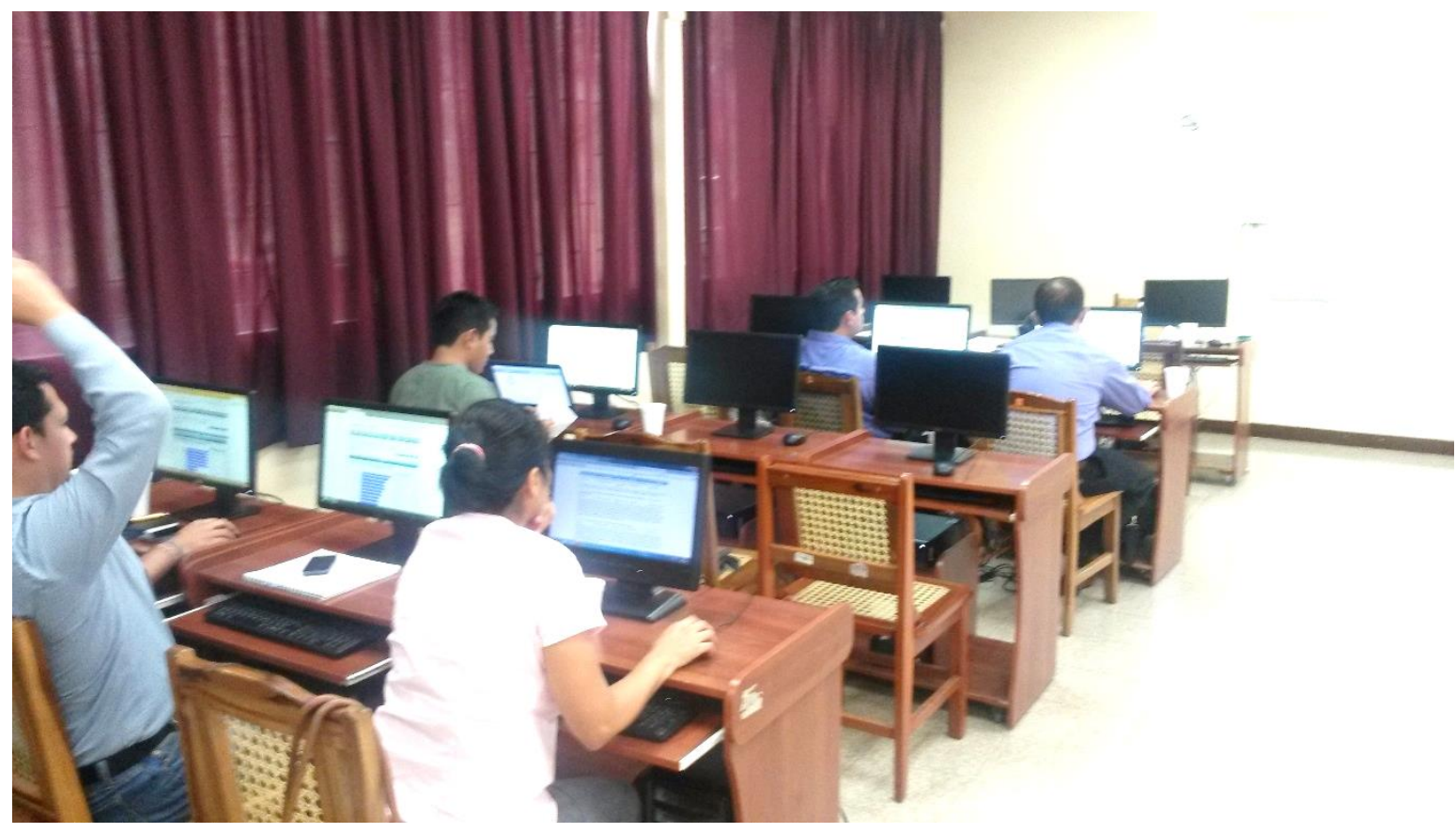

Figura 4. Coordinadores del CATI de facultades, IPS y centros de Investigación haciendo ejercicios de búsqueda en PATENTSCOPE.

Las bases de Datos Consultadas fueron:

- PATENTSCOPE 


\section{- ESPACENET.}

\section{Resultados obtenidos.}

Asistencia técnica a las oficinas de enlace por medio de Las Tecnologías de Información y Comunicación, relacionadas con los procesos de la instalación de los kioskos informáticos.

Asesoramiento a los coordinadores de la oficina de enlace de los CATI's, en el uso de las bases de Datos de ESPACENET y PATENTSCOPE.

Asistencia en: Transferencia de Tecnologías; Adaptación de la tecnología de las bases de Datos; Procesos concernientes en la protección de los derechos de la propiedad intelectual en las creaciones desarrolladas por inventores, obtenedores y autores de UNAN - Managua, ante el registro de Propiedad Intelectual de la República de Nicaragua; Leyes rectoras de la República de Nicaragua sobre la protección de los derechos de Propiedad Intelectual de Nicaragua; Materias excluidas de Patente; Procedimientos sobre los procesos de Protección de la Propiedad Intelectual de Nicaragua; Conocimiento General de la Propiedad Intelectual. 